\title{
An isolated ruptured spinal aneurysm presents with a thalamic Infarct: case report
}

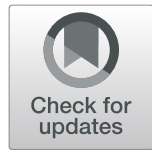

Alexander Tenorio ${ }^{1 *}$, Brandon B. Holmes ${ }^{1}$, Adib A. Abla², Matthew Amans ${ }^{3}$ and Karl Meisel ${ }^{1}$

\begin{abstract}
Background: Isolated spinal artery aneurysms are extremely rare, and their pathogenesis, clinical presentation, and treatment strategies are poorly established. We report only the second case of a patient with an isolated posterior spinal aneurysm and concurrent left thalamic infarct and review the literature to help clarify treatment strategies of isolated spinal aneurysms.

Case presentation: A 49-year-old patient presented with acute onset walking difficulty followed by diaphoresis, back and abdominal pain, and paraplegia. Imaging was notable for a hemorrhagic spinal lesion with compression at T12 through $L 4$ and an acute left thalamic infarct. Surgical exploration revealed an isolated posterior spinal artery aneurysm. The aneurysm was surgically resected and the patient had partial recovery six months post-operatively.

Conclusions: Isolated posterior spinal artery aneurysms of the thoracolumbar region are rare lesions that commonly present with abdominal pain, radiating back pain, and lower extremity weakness. Imaging may not provide a definitive diagnosis. The three primary treatment strategies are conservative management, endovascular treatment, or surgical resection. In patients with symptomatic cord compression, immediate surgical intervention is indicated to preserve neurologic function. In all other cases, the artery size, distal flow, morphology, and location may guide management.
\end{abstract}

Keywords: Posterior spinal aneurysm, Subarachnoid hemorrhage, Thalamic infarct

\section{Background}

Spinal artery aneurysms are a rare condition typically associated with vascular anomalies that generate local flow alterations such as fistulas, arteriovenous malformations (AVM), and coarctation of the aorta. When found in the absence of these vascular pathologies, they are referred to as isolated spinal artery aneurysms and usually occur in the anterior spinal artery. Isolated spinal artery aneurysms arising from the posterior axis of the spinal cord are exceptionally rare with optimal medical and surgical management being poorly defined. We report a case of a patient with a spinal subarachnoid hemorrhage caused by rupture of an isolated posterior spinal artery aneurysm that was surgically corrected. The patient also

\footnotetext{
* Correspondence: alexander.tenorio@ucsf.edu

'Department of Neurology, University of California, San Francisco, CA, USA

Full list of author information is available at the end of the article
}

presented with a concurrent thalamic infarct which has only been described in the literature once before [1].

\section{Case presentation \\ Clinical findings}

A 49-year-old patient with a history of vestibular schwannoma and epilepsy developed acute onset walking difficulty. Two days later, the patient presented to an outside hospital with diaphoresis, abdominal pain, back pain radiating to the thighs, and bilateral lower extremity paralysis. A brain MRI revealed an acute left thalamic infarct and a spine MRI found compression at T12 through L4 concerning for subarachnoid or subdural hemorrhage. The patient was transferred to our institution for further work-up and management. On our admission, examination revealed dense lower extremity paraplegia with anesthesia and absent rectal tone

(c) The Author(s). 2021 Open Access This article is licensed under a Creative Commons Attribution 4.0 International License, which permits use, sharing, adaptation, distribution and reproduction in any medium or format, as long as you give appropriate credit to the original author(s) and the source, provide a link to the Creative Commons licence, and indicate if changes were made. The images or other third party material in this article are included in the article's Creative Commons licence, unless indicated otherwise in a credit line to the material. If material is not included in the article's Creative Commons licence and your intended use is not permitted by statutory regulation or exceeds the permitted use, you will need to obtain permission directly from the copyright holder. To view a copy of this licence, visit http://creativecommons.org/licenses/by/4.0/ The Creative Commons Public Domain Dedication waiver (http://creativecommons.org/publicdomain/zero/1.0/) applies to the data made available in this article, unless otherwise stated in a credit line to the data. 
concerning for conus medullaris syndrome. Additionally, the patient had right hemi-body sensory loss of light touch and pinprick in the face, upper torso, and arm.

\section{Imaging}

MRI with MRA of the complete spine demonstrated an intradural cystic and hemorrhagic mass along the left ventrolateral pial surface at the T11-12 level. This lesion was associated with extensive intradural hemorrhage with inferior extension into the lumbar spine (Fig. 1). The imaging findings were highly suggestive of subarachnoid and subdural hemorrhage initially thought to be due to a cavernous malformation vs. AVM. Brain MRI illustrated a T2 FLAIR hyperintense signal within the left thalamus consistent with an acute thalamic infarct. Conventional spinal angiography was negative for evidence of spinal aneurysm.

\section{Surgical Approach and Pathology}

The following day, a laminectomy was performed at levels T10- L1. The dura was opened and blood was immediately visualized and evacuated from the subdural space. The dura was retracted laterally with subarachnoid blood becoming apparent. The arachnoid was opened and the subarachnoid blood was irrigated and removed using suction. At the left lateral aspect of T11 and T12, there was a hematoma compressing the spinal cord in the subdural space, which was also evacuated with suction. After removal of the hematoma, a nodular vascular lesion at the posterolateral spinal cord likely emanating from the posterolateral spinal artery was visualized, excised, and sent to pathology (Fig. 1c).

Surgical pathology demonstrated a thin segment of vessel wall, positive trichrome stain, and several peripheral reticulin fibers confirming the diagnosis of a spinal aneurysm (Fig. 1d). Post-operatively, the patient had improved sensation and gradually regained strength in the lower extremities. The patient was discharged to the transferring hospital five days after surgery. Six months post-operatively, the patient's sensation and strength had improved but remained unable to walk with movement in the lower extremities limited to the toes.

\section{Left thalamic infarct}

The left thalamic infarct was treated with standard poststroke management. The patient was kept permissively
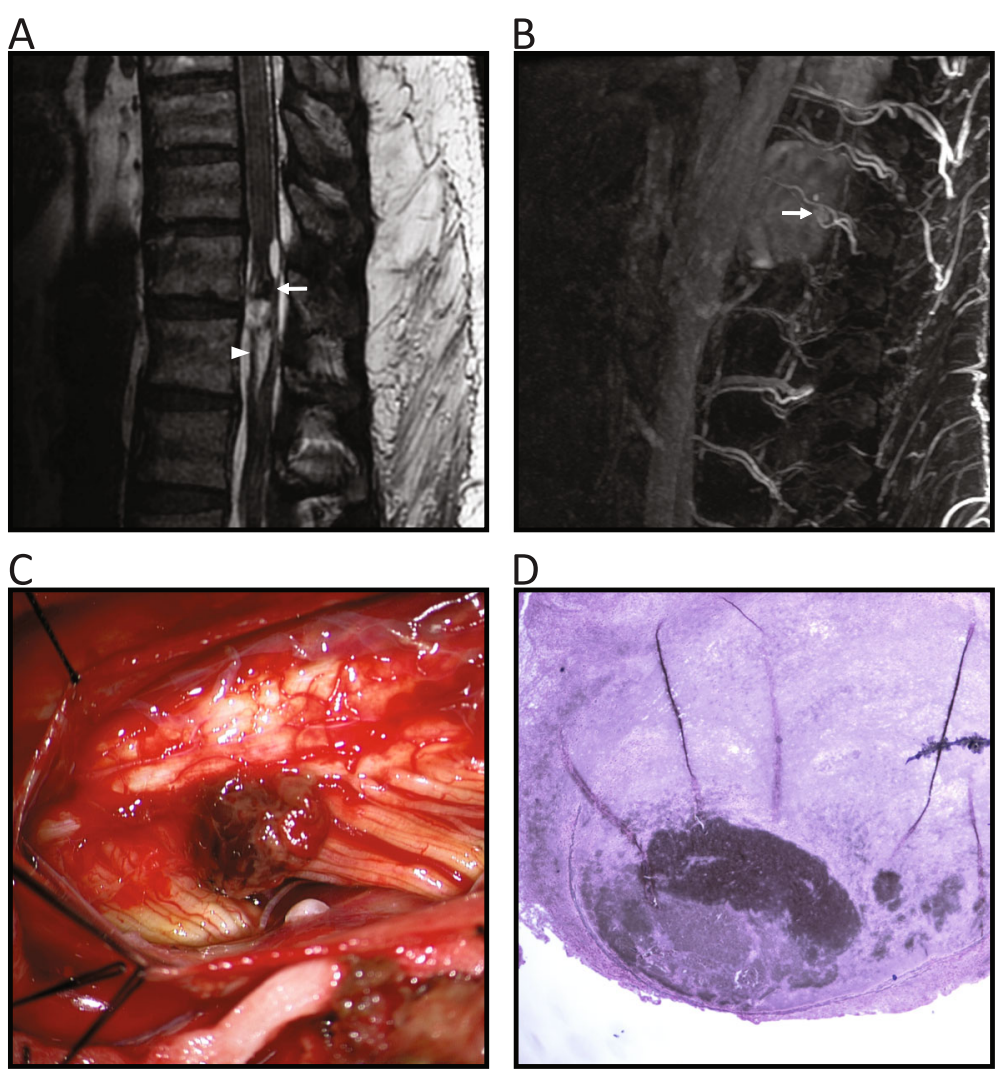

Fig. 1 a Balanced steady-state gradient recalled echo sequences demonstrate a $1.7 \mathrm{~cm}$ intradural cystic and hemorrhagic mass within the spinal cord at thoracic levels 11 and 12 (arrow). There is associated intradural hemorrhage (arrowhead). b Spinal magnetic resonance angiogram shows a saccular aneurysm (arrow). c Surgical photograph reveals the posterior spinal aneurysm as an extrinsic mass lesion. $\mathbf{d}$ Histopathology of the resected aneurysm demonstrates positive reticulin staining 
hypertensive and Atorvastatin was initiated. HgbAlc was within normal range and Aspirin was started on postoperative day number 5; DAPT was not initiated given spinal SAH. The patient underwent a transthoracic echocardiogram and MRA of the neck, which were both unremarkable. The hemi-body sensory loss gradually improved and sensation was back to near baseline at discharge five days later.

\section{Discussion and conclusion}

We present a case of a ruptured posterior spinal artery aneurysm with a concurrent left thalamic infarct. This combination of pathologies led to cauda equina syndrome with hemibody sensory loss. To our knowledge, this is the second reported case of an isolated spinal artery aneurysm associated with a left thalamic infarct.

The pathophysiology of isolated spinal artery aneurysms is still under investigation. The majority are associated with vascular malformations, with increased flow through the vessel leading to aneurysm formation [2]. In the absence of a malformation (i.e. isolated spinal aneurysms), dissection has been a proposed mechanism proven by histopathology [3]. These dissections often arise in the setting of a condition that weakens the vessels, such as connective tissue disorders and autoimmune disease [2]. In our case, surgical examination revealed a ruptured thrombosed aneurysm without evidence of dissection on histopathology.

The natural history of isolated spinal aneurysms is unclear due to its rarity. Through a literature review, we found 39 cases of isolated spinal aneurysms from 2011 to 2019 (Table 1). Kim et al. previously found 43 cases as of 2010 [3], bringing the total number to 83 when including our case. Our patient initially presented with radiating back pain, abdominal pain, and lower extremity weakness. This is consistent with previous reports, as the most common symptoms in thoracolumbar spinal aneurysms are sudden onset back pain, weakness, meningism, and abdominal pain [3]. A hemorrhagic lesion was also present on initial imaging, which was confirmed to be a ruptured aneurysm on surgical examination. Hemorrhage occurs at a very high rate in patients with spinal aneurysms associated with AVMs, with one review finding a $100 \%$ incidence in 12 patients [4]. Although the data for isolated spinal aneurysms is limited, they are also likely to present with rupture and hemorrhage. In Kim et. al.'s review, 36 (84\%) presented with rupture [3].

The treatment strategy for spinal aneurysms is also controversial, with three primary strategies being used: (1) surgical resection, (2) endovascular treatment, or (3) conservative management [3]. Some authors have advocated for conservative management given their experience of spontaneous regression [5, 6]. They suggest that compression of the spinal cord from the aneurysm or surrounding blood should be the only indications for intervention. Karakama et al. presented a case of a ruptured anterior spinal artery aneurysm treated conservatively and recommended strict blood pressure control and follow-up imaging to monitor for progression [7]. This aneurysm was located in the anterior cervical region and conservative management was selected due to concern of disturbing blood flow of the parent artery. Longatti et al. presented a case of a patient with multiple anterior spinal artery aneurysms that were treated conservatively [8]. This strategy was selected due to the absence of cord compression, small artery size, and presence of distal flow. Dabus et al. presented a case series of four patients with dissecting spinal aneurysms that were treated conservatively, with the size of the parent artery and tissue supplied being the main determinants [9]. In these cases, the artery size, presence of distal flow, fusiform morphology, and surgical access were factors that favored a conservative approach.

Despite the successful cases with a wait-and-see approach, the overall occurrence of this condition is too limited to draw definite conclusions. There have been five reported cases of a patient dying without surgical intervention, with re-bleeding being the most common cause [10-14]. Early surgical intervention has been proposed for posterior spinal aneurysms due to its superficial location and ability to be resected safely, with all cases resulting in complete resolution [15]. Anterior spinal aneurysms are more difficult to access surgically and provide major blood supply to the spinal cord, which can lead to severe neurologic sequalae if interrupted. In our case, the patient presented with progressive symptoms of spinal cord compression, most notably paraplegia. Imaging did not provide a definitive diagnosis, so prompt surgical intervention was indicated to decompress the spinal cord and preserve neurologic function.

Whether the spinal aneurysm was the source of the left thalamic infarct or an incidental finding is difficult to determine. To the best of our knowledge, no cases in the literature have reported a spinal aneurysm as a cause of a cerebral infarct, although one case exists linking a subarachnoid hemorrhage with a spinal cord infarct [16]. This suggests that blood products in the subarachnoid space might lead to secondary ischemia at a distance, and indeed, multiple mechanisms have been postulated that connect subarachnoid blood with micro and macro circulatory failure. For example, release of intracellular material such as oxyhemoglobin from red blood cell lysis can lead to altered vessel dynamics through inactivation of nitric oxide, over-expression of endothelin peptides, and under-expression of 
Table 1 Demographic and Clinical Data of Isolated Spinal Aneurysms (2011-2019)

\begin{tabular}{|c|c|c|c|c|c|c|c|c|}
\hline No. & $\begin{array}{l}\text { Author/ } \\
\text { Year }\end{array}$ & Age/Sex & Co-morbidities & Location & Initial Presentation & Imaging Findings & Treatment & Outcome \\
\hline 1 & $\begin{array}{l}\text { lihoshi } \\
2011[17]\end{array}$ & $60 / F$ & - & $\mathrm{T} 11$ & $\begin{array}{l}\text { Headache, back pain, } \\
\text { nausea }\end{array}$ & $\begin{array}{l}\text { Spinal and } \\
\text { Intracranial SAH }\end{array}$ & Conservative & Resolution \\
\hline 2 & $\begin{array}{l}\text { Kim } 2012 \\
{[3]}\end{array}$ & $52 / \mathrm{M}$ & $\begin{array}{l}\text { Right acoustic } \\
\text { neuroma, HTN, } \\
\text { Meningoencephalitis }\end{array}$ & $\mathrm{T7}$ & $\begin{array}{l}\text { Abdominal pain, } \\
\text { headache, back pain } \\
\text { radiating to LE }\end{array}$ & $\begin{array}{l}\text { T7 Intradural } \\
\text { extramedullary } \\
\text { enhancing lesion }\end{array}$ & Embolization & Resolution \\
\hline 3 & $\begin{array}{l}\text { Shankar } \\
2012[18]\end{array}$ & $72 / F$ & - & L2 & Back pain radiating to LE & T12-L1 Lesion & Embolization & Improved \\
\hline 4 & $\begin{array}{l}\text { Takashima } \\
2012[19]\end{array}$ & $84 / \mathrm{M}$ & - & $\mathrm{C} 1$ & Quadriplegia & $\begin{array}{l}\text { Intramedullary C1 } \\
\text { Hematoma }\end{array}$ & $\mathrm{N} / \mathrm{A}$ & $\begin{array}{l}\text { Death from } \\
\text { respiratory } \\
\text { dysfunction }\end{array}$ \\
\hline 5 & $\begin{array}{l}\text { Tanweer } \\
2012[20]\end{array}$ & $67 / F$ & HTN, Atrial Fibrillation & T11 & $\begin{array}{l}\text { Back pain, Acute } \\
\text { paraplegia and sensory } \\
\text { loss }\end{array}$ & $\begin{array}{l}\text { Spinal and } \\
\text { Intracranial SAH }\end{array}$ & Embolization & Improved \\
\hline 6 & $\begin{array}{l}\text { Seerangan } \\
2012[21]\end{array}$ & $47 / M$ & $\begin{array}{l}\text { Intracranial aneurysms, } \\
\text { ESRD, ADPKD }\end{array}$ & T7-Т10 & $\begin{array}{l}\text { Lower extremity } \\
\text { weakness, bowel/bladder } \\
\text { disturbances }\end{array}$ & $\begin{array}{l}\text { Intracranial and } \\
\text { Spinal SAH }\end{array}$ & Resection & $\begin{array}{l}\text { Minimal } \\
\text { Recovery }\end{array}$ \\
\hline 7 & $\begin{array}{l}\text { Sato } 2012 \\
{[22]}\end{array}$ & $67 / F$ & HTN, Dyslipidemia & $\begin{array}{l}\text { T8 and } \\
\text { T10 }\end{array}$ & $\begin{array}{l}\text { Acute back pain, } \\
\text { paraparesis }\end{array}$ & $\begin{array}{l}\text { T8 and T10 intradural } \\
\text { masses, spinal } \\
\text { infarction, spinal SAH }\end{array}$ & Conservative & Resolution \\
\hline 8 & $\begin{array}{l}\text { Van Es } \\
2013[23]\end{array}$ & $62 / F$ & None & $\mathrm{T} 12$ & $\begin{array}{l}\text { Headache, back pain, } \\
\text { walking difficulty }\end{array}$ & Spinal SAH & Resection & - \\
\hline 9 & $\begin{array}{l}\text { Van Es } \\
2013[23]\end{array}$ & $68 / M$ & - & T4 & $\begin{array}{l}\text { Intrascapular back pain } \\
\text { radiating to lumbar } \\
\text { region, Headache, Nausea }\end{array}$ & $\begin{array}{l}\text { T4 Hyperdense } \\
\text { nodular lesion }\end{array}$ & $\begin{array}{l}\text { Conservative } \\
\text { (patient } \\
\text { refusal) }\end{array}$ & Resolution \\
\hline 10 & $\begin{array}{l}\text { Marovich } \\
2013[24]\end{array}$ & $58 / \mathrm{M}$ & None & & $\begin{array}{l}\text { Cervico-thoracic back } \\
\text { pain }\end{array}$ & $\begin{array}{l}\text { C8-T6 Extradural } \\
\text { Hemorrhagic Lesion }\end{array}$ & Resection & - \\
\hline 11 & $\begin{array}{l}\text { Yang } 2013 \\
{[25]}\end{array}$ & $47 / M$ & - & $\begin{array}{l}\text { ASA- } \\
\text { cervical } \\
\text { region }\end{array}$ & Neck Pain & $\begin{array}{l}\text { Cranial SAH, IVH, ASA } \\
\text { aneurysm }\end{array}$ & Conservative & $\begin{array}{l}\text { Death from } \\
\text { End-Stage Bile } \\
\text { Duct Cancer }\end{array}$ \\
\hline 12 & $\begin{array}{l}\text { Son } 2013 \\
{[26]}\end{array}$ & $45 / F$ & None & L1 & $\begin{array}{l}\text { Headache, back pain, } \\
\text { nausea }\end{array}$ & $\begin{array}{l}\text { Spinal and } \\
\text { Intracranial SAH }\end{array}$ & Conservative & Resolution \\
\hline 13 & $\begin{array}{l}\text { Santana- } \\
\text { Ramirez } \\
2013[27]\end{array}$ & $1 / F$ & - & $\mathrm{C} 3-\mathrm{C} 6$ & Quadriparesis, neck pain & $\begin{array}{l}\text { C3-C6 intramedullary } \\
\text { lesion }\end{array}$ & Resection & Improved \\
\hline 14 & $\begin{array}{l}\text { Pahl } 2014 \\
{[28]}\end{array}$ & $43 / F$ & None & $\begin{array}{l}\text { Cervi- } \\
\text { medullary } \\
\text { junction }\end{array}$ & Headache and vomiting & Intracranial SAH, IVH & Conservative & Resolution \\
\hline 15 & $\begin{array}{l}\text { Romero } \\
2014 \text { [29] }\end{array}$ & $37 / F$ & - & $\mathrm{T} 4$ & $\begin{array}{l}\text { Thoracic/cervical pain, } \\
\text { headache }\end{array}$ & $\begin{array}{l}\text { Spinal and } \\
\text { Intracranial SAH }\end{array}$ & Conservative & Resolution \\
\hline 16 & $\begin{array}{l}\text { Romero } \\
2014 \text { [29] }\end{array}$ & $72 / F$ & HTN, DM, CRF & $\mathrm{T} 10$ & $\begin{array}{l}\text { Cervical pain, headache, } \\
\text { neck stiffness }\end{array}$ & $\begin{array}{l}\text { Spinal and } \\
\text { Intracranial SAH }\end{array}$ & Conservative & Improved \\
\hline 17 & $\begin{array}{l}\text { Bell } 2014 \\
{[30]}\end{array}$ & $68 / F$ & - & T5 & Severe back pain & $\begin{array}{l}\text { Thoracic intradural } \\
\text { lesion and lumbar } \\
\text { SAH }\end{array}$ & Resection & - \\
\hline 18 & $\begin{array}{l}\text { Johnson } \\
2015 \text { [31] }\end{array}$ & Teenager/- & None & $\mathrm{C} 5-\mathrm{C} 6$ & $\begin{array}{l}\text { Headache, neck pain, } \\
\text { nausea }\end{array}$ & $\begin{array}{l}\text { C5-C6 enhancing } \\
\text { nodular lesion }\end{array}$ & Resection & Resolution \\
\hline 19 & $\begin{array}{l}\text { Ronchetti } \\
2015 \text { [32] }\end{array}$ & $51 / F$ & - & T1-T4 & $\begin{array}{l}\text { Neck pain, headache, } \\
\text { bilateral leg numbness, } \\
\text { difficulty walking }\end{array}$ & $\begin{array}{l}\text { Thoracic } \\
\text { extramedullary } \\
\text { hemorrhage }\end{array}$ & Resection & Resolution \\
\hline 20 & $\begin{array}{l}\text { Ronchetti } \\
2015 \text { [32] }\end{array}$ & $68 / M$ & - & $\mathrm{T} 1$ & $\begin{array}{l}\text { Mid-back pain radiating } \\
\text { to neck }\end{array}$ & $\begin{array}{l}\text { Intracranial SAH, } \\
\text { Cervico-Thoracic SAH }\end{array}$ & Embolization & Resolution \\
\hline 21 & $\begin{array}{l}\text { Sung } 2015 \\
{[33]}\end{array}$ & $74 / \mathrm{M}$ & $\begin{array}{l}\text { HTN, Ischemic Heart } \\
\text { Disease }\end{array}$ & $\mathrm{T} 1$ & $\begin{array}{l}\text { Chest pain radiating to } \\
\text { neck/back }\end{array}$ & $\begin{array}{l}\text { Intracranial and } \\
\text { Spinal SAH }\end{array}$ & Resection & Resolution \\
\hline 22 & Horio 2015 & $84 / \mathrm{M}$ & Right Thalamic Infarct & $\mathrm{T} 12$ & Left Hemiplegia & Spinal SAH & Resection & Improved \\
\hline
\end{tabular}


Table 1 Demographic and Clinical Data of Isolated Spinal Aneurysms (2011-2019) (Continued)

\begin{tabular}{|c|c|c|c|c|c|c|c|c|}
\hline No. & $\begin{array}{l}\text { Author/ } \\
\text { Year }\end{array}$ & Age/Sex & Co-morbidities & Location & Initial Presentation & Imaging Findings & Treatment & Outcome \\
\hline 23 & $\begin{array}{l}\text { Takata } \\
2016 \text { [34] }\end{array}$ & $72 / F$ & None & T9 & Acute back pain & T4-T10 SAH & Resection & Resolution \\
\hline 24 & $\begin{array}{l}\text { Doberstein } \\
2016 \text { [35] }\end{array}$ & $59 / \mathrm{M}$ & $\begin{array}{l}\text { Parkinson's, T-cell } \\
\text { lymphoma }\end{array}$ & $\mathrm{T} 11$ & $\begin{array}{l}\text { Back spasms, walking } \\
\text { difficulty }\end{array}$ & T6-L2 Hyperintensity & Conservative & Resolution \\
\hline 25 & $\begin{array}{l}\text { Ikeda } 2016 \\
\text { [36] }\end{array}$ & $54 / \mathrm{M}$ & - & $\mathrm{T} 10$ & $\begin{array}{l}\text { Severe back pain, } \\
\text { vomiting }\end{array}$ & Spinal SDH and SAH & Resection & Resolution \\
\hline 26 & $\begin{array}{l}\text { Hill } 2016 \\
{[37]}\end{array}$ & $53 / \mathrm{M}$ & $\mathrm{HBV}, \mathrm{HCV}$ & T9 & Paraplegia & $\begin{array}{l}\text { C7-T1 intradural } \\
\text { lesion }\end{array}$ & Resection & $\begin{array}{l}\text { Death from } \\
\text { medical } \\
\text { complications }\end{array}$ \\
\hline 27 & $\begin{array}{l}\text { Roka } 2016 \\
\text { [38] }\end{array}$ & $30 / F$ & None & $\begin{array}{l}\text { Cervical } \\
\text { ASA }\end{array}$ & $\begin{array}{l}\text { Headache, vomiting, } \\
\text { vertigo }\end{array}$ & IVH & Conservative & Resolution \\
\hline 28 & $\begin{array}{l}\text { Kogan } \\
2017 \text { [39] }\end{array}$ & $58 / F$ & - & $\mathrm{T} 2$ & $\begin{array}{l}\text { Headache radiating to } \\
\text { neck and upper back, } \\
\text { nausea, vomiting }\end{array}$ & T1-T5 hyperintensity & Resection & Resolution \\
\hline 29 & $\begin{array}{l}\text { Dabus } \\
2018[9]\end{array}$ & $60 \mathrm{~s} /-$ & - & $\begin{array}{l}\text { Cervico- } \\
\text { medullary } \\
\text { Junction }\end{array}$ & Headache and neck pain & SAH & Conservative & Resolution \\
\hline 30 & $\begin{array}{l}\text { Dabus } \\
2018[9]\end{array}$ & $30 \mathrm{~s} /-$ & - & Cervical & Back Pain & SAH & Conservative & Resolution \\
\hline 31 & $\begin{array}{l}\text { Dabus } \\
2018[9]\end{array}$ & $60 \mathrm{~s} /-$ & - & $\begin{array}{l}\text { Mid- } \\
\text { Thoracic }\end{array}$ & $\begin{array}{l}\text { Back pain and LE } \\
\text { paresthesia }\end{array}$ & $\begin{array}{l}\text { Intramedullary } \\
\text { Hemorrhage }\end{array}$ & Conservative & Resolution \\
\hline 32 & $\begin{array}{l}\text { Dabus } \\
2018[9]\end{array}$ & $50 \mathrm{~s} /-$ & - & $\begin{array}{l}\text { Lower } \\
\text { Thoracic }\end{array}$ & $\begin{array}{l}\text { Back pain and LE } \\
\text { paresthesia }\end{array}$ & $\mathrm{SDH}$ & Conservative & Resolution \\
\hline 33 & $\begin{array}{l}\text { Aljuboori } \\
2018[40]\end{array}$ & $78 / \mathrm{M}$ & HTN, PVD, HLD, CAD & T9 & $\begin{array}{l}\text { Acute Back Pain, LE } \\
\text { weakness }\end{array}$ & $\begin{array}{l}\text { T9 aneurysm, cord } \\
\text { compression }\end{array}$ & Resection & Resolution \\
\hline 34 & $\begin{array}{l}\text { Aguilar- } \\
\text { Salinas } \\
2018[41]\end{array}$ & $54 / F$ & HTN & $\mathrm{T} 10$ & $\begin{array}{l}\text { Headache, back pain, } \\
\text { nausea, vomiting }\end{array}$ & $\begin{array}{l}\text { Spinal SAH with cord } \\
\text { compression }\end{array}$ & Conservative & Improved \\
\hline 35 & $\begin{array}{l}\text { Ren } 2018 \\
{[42]}\end{array}$ & $57 / F$ & N/A & $\mathrm{C} 1$ & Severe headache & Intracranial SAH & Resection & Resolution \\
\hline 36 & $\begin{array}{l}\text { Ren } 2018 \\
{[42]}\end{array}$ & $27 / F$ & N/A & L1 & $\begin{array}{l}\text { Bilateral LE pain/ } \\
\text { numbness }\end{array}$ & $\begin{array}{l}\text { Lesion at Conus } \\
\text { Medullaris }\end{array}$ & Resection & Resolution \\
\hline 37 & $\begin{array}{l}\text { Morozumi } \\
2018[43]\end{array}$ & $9 / M$ & None & C7-T1 & Back pain, paralysis & $\begin{array}{l}\text { C7-T1 Lesion with } \\
\text { hemorrhage }\end{array}$ & Resection & Resolution \\
\hline 38 & $\begin{array}{l}\text { Simon- } \\
\text { Gabriel } \\
2018[44]\end{array}$ & $65 / M$ & $\begin{array}{l}\text { HTN, } \\
\text { Hypercholesterolemia, } \\
\text { Tachyarrhythmia }\end{array}$ & $\begin{array}{l}\text { Cranio- } \\
\text { cervical } \\
\text { ASA }\end{array}$ & Neck stiffness & $\begin{array}{l}\text { SAH with } \\
\text { tamponade of } 4 \text { th } \\
\text { ventricle }\end{array}$ & $\begin{array}{l}\text { Flow } \\
\text { diverting } \\
\text { stent }\end{array}$ & Resolution \\
\hline 39 & $\begin{array}{l}\text { Priola } 2019 \\
\text { [45] }\end{array}$ & $54 / F$ & None & $\mathrm{T} 3$ & $\begin{array}{l}\text { Upper thoracic back pain } \\
\text { radiating to neck and } \\
\text { head }\end{array}$ & $\begin{array}{l}\text { Cervico-thoraco- } \\
\text { lumbar spine } \\
\text { Hematomas }\end{array}$ & Resection & Resolution \\
\hline
\end{tabular}

*HTN Hypertension, DM Diabetes Mellitus, CRF Chronic Renal Failure, ESRD End Stage Renal Disease, ADPKD Autosomal Dominant Polycystic Kidney Disease, PVD Peripheral Vascular Disease, HLD Hyperlipidemia, CAD Coronary Artery Disease, HBV Hepatitis B Virus, HCV Hepatitis C Virus, LE Lower Extremities, SAH Subarachnoid Hemorrhage, IVH Intraventricular Hemorrhage, ASA Anterior Spinal Artery

Search Strategy:

- Google scholar: isolated AND spinal AND aneurysm

- Date range: 2011-2019

- 990 results reviewed

prostacyclin leading to platelet aggregation [17]. Should these mechanisms have contributed to the thalamic infarct observed in our patient, it is unclear why the arteries supplying the thalamus would have been particularly susceptible. Along these lines, MRA of the head and neck did not reveal evidence of vasculopathy. An additional mechanism to link these two pathologies is hypertension. Hypertension is common following subarachnoid hemorrhage likely due to pain, anxiety, and sympathetic activation. Thalamic infarcts are most often caused by microvascular disease with hypertension accounting for $\sim 68 \%$ of cases [18]. It is noteworthy that in the 83 cases of isolated spinal artery aneurysms reported in literature, two cases with concurrent thalamic infarcts have now been observed which is statistically unexpected. Further investigations are needed to 
determine whether there is a mechanistic link between these two pathologies, and future cases may benefit by pursuing cerebral angiography at the same time of spinal angiography.

In conclusion, isolated spinal artery aneurysms are an exceedingly rare occurrence, particularly in the posterior axis of the spine. Imaging with MRI/CT and angiogram may not provide a definitive diagnosis. We agree with previous authors that in a patient with a posterior spinal aneurysm and symptoms of spinal cord compression, prompt surgical intervention is warranted. Finally, further studies are needed to understand the possible interaction between subarachnoid blood products and cerebral infarction.

\section{Acknowledgements}

Not applicable.

\section{Authors' contributions}

AT drafted and revised the manuscript. BH, AA, MA, and KM provided images and edited/reviewed the manuscript. All authors read and approved the final manuscript.

\section{Funding}

Not applicable.

\section{Availability of data and materials}

All data generated or analyzed during this study are included in this published article and its supplementary information files.

\section{Ethics approval and consent to participate}

Not applicable.

\section{Consent for publication}

Patient has read and reviewed the manuscript and given written consent for publication.

\section{Competing interests}

The authors declare that they have no competing interests.

\section{Author details}

${ }^{1}$ Department of Neurology, University of California, San Francisco, CA, USA. ${ }^{2}$ Department of Neurosurgery, University of California, San Francisco, CA, USA. ${ }^{3}$ Department of Neurointerventional Radiology, University of California, San Francisco, CA, USA.

Received: 29 January 2020 Accepted: 12 January 2021

Published online: 03 February 2021

\section{References}

1. Horio Y. Katsuta T, Samura K, et al. Successfully Treated Isolated Posterio Spinal Artery Aneurysm Causing Intracranial Subarachnoid Hemorrhage: Case Report. Neurol Med Chir (Tokyo). 2015:55(12):915-9.

2. Renieri L. Raz E, Lanzino G, et al. Spinal artery aneurysms: clinical presentation, radiological findings and outcome. J Neurointerv Surg. 2018; 10(7):644-8.

3. Kim HJ. Choi IS. Dissecting aneurysm of the posterior spinal artery: case report and review of the literature. Neurosurgery. 2012;71(3):E749-56.

4. Massand MG. Wallace RC, Gonzalez LF. Zabramski JM, Spetzler RF. Subarachnoid hemorrhage due to isolated spinal artery aneurysm in four patients. AJNR Am J Neuroradiol. 2005;26(9):2415-9.

5. Berlis A. Scheufler KM, Schmahl C. Rauer S, Götz F. Schumacher M. Solitary spinal artery aneurysms as a rare source of spinal subarachnoid hemorrhage: potential etiology and treatment strategy. AJNR Am J Neuroradiol. 2005;26(2):405-10.
6. Berlis A. Schumacher M. Subarachnoid hemorrhage due to isolated spinal arteries: Rare cases with controversy about the treatment strategy. AJNR Am J Neuroradiol. 2006;27(4):726-7.

7. Karakama J. Nakagawa K, Maehara T. Ohno K. Subarachnoid hemorrhage caused by a ruptured anterior spinal artery aneurysm. Neurol Med Chir (Tokyo). 2010;50(11):1015-9.

8. Longatti P. Sgubin D, Di paola F. Bleeding spinal artery aneurysms. J Neurosurg Spine. 2008;8(6):574-8.

9. Dabus G. Tosello RT, Pereira BJA. Linfante I, Piske RL. Dissecting spinal aneurysms: conservative management as a therapeutic option. J Neurointerv Surg. 2018;10(5):451-4.

10. Garcia CA. Dulcey S, Dulcey J. Ruptured aneurysm of the spinal artery of Adamkiewicz during pregnancy. Neurology. 1979;29(3):394-8.

11. Koçak A. Ateş O, Cayli SR. Saraç K. Isolated posterior spinal artery aneurysm. Br J Neurosurg. 2006:20(4):241-4.

12. Yonas H. Patre S, White RJ. Anterior spinal artery aneurysm. Case report. J Neurosurg. 1980:53(4):570-3.

13. Henson RA. Croft PB. Spontaneous spinal subarachnoid haemorrhage. Q J Med. 1956;25(97):53-66.

14. Rengachary SS. Duke DA, Tsai FY. Kragel PJ. Spinal arterial aneurysm: case report. Neurosurgery. 1993:33(1):125-9.

15. Geibprasert S. Krings T, Apitzsch J. Reinges MH, Nolte KW. Hans FJ. Subarachnoid hemorrhage following posterior spinal artery aneurysm. A case report and review of the literature. Interv Neuroradiol. 2010;16(2):183-90.

16. Krishna V. Lazaridis C, Ellegala D. Galzier S, Kindy M. Spampinato J, Chalela JA. Spinal cord infarction associated with subarachnoid hemorrhage. Clin Neurol Neurosug. 2012;114(7):1030-2.

17. Khurana VG. Besser M. Pathophysiological basis of cerebral vasospasm following aneurysmal subarachnoid haemorrhage. J Clin Neurosci. 1997;4(2):122-31.

18. Kumral E. Evyapan D, Kutluhan S. Pure thalamic infarctions: Clinical findings. J Stroke Cerebrovasc Dis. 2000;9(6):287-97.

19. lihoshi S. Miyata K, Murakami T. Kaneko T, Koyanagi I. Dissection aneurysm of the radiculomedullary branch of the artery of Adamkiewicz with subarachnoid hemorrhage. Neurol Med Chir (Tokyo). 2011;51(9):649-52.

20. Shankar JJ. Terbrugge K, Krings T. Subarachnoid hemorrhage following posterior spinal artery aneurysm rupture. Can J Neurol Sci. 2012;39(4):531-2.

21. Takashima N. Murai H, Hirano S. Oya M. Isolated intramedullary spinal artery aneurysm. Neurology. 2012;79(6):608-9.

22. Tanweer O. Woldenberg R, Zwany S. Setton A. Endovascular obliteration of a ruptured posterior spinal artery pseudoaneurysm. J Neurosurg Spine. 2012;17(4):334-6.

23. Seerangan G. Narayanan M. A Rare Case of Subarachnoid Hemorrhage due to Rupture of Isolated Anterior Spinal Artery Aneurysm in a Patient with Polycystic Kidney Disease. Case Rep Nephrol Urol. 2012;2(2):108-12.

24. Sato K. Roccatagliata L, Depuydt S. Rodesch G. Multiple aneurysms of thoracic spinal cord arteries presenting with spinal infarction and subarachnoid hemorrhage: case report and literature review. Neurosurgery. 2012;71(5):E1053-8.

25. Van es AC. Brouwer PA, Willems PW. Management considerations in ruptured isolated radiculopial artery aneurysms. A report of two cases and literature review. Interv Neuroradiol. 2013;19(1):60-6.

26. Marovic P. Thani N, Lu S. Bala A. Spinal subarachnoid hemorrhage secondary to rupture of an isolated radicular artery aneurysm. J Neurol Surg A Cent Eur Neurosurg. 2013;74(6):410-4.

27. Yang TK. A ruptured aneurysm in the branch of the anterior spinal artery. J Cerebrovasc Endovasc Neurosurg. 2013;15(1):26-9.

28. Son S. Lee SG, Park CW. Solitary ruptured aneurysm of the spinal artery of adamkiewicz with subarachnoid hemorrhage. J Korean Neurosurg Soc. 2013:54(1):50-3.

29. Santana-Ramírez A. Farias-serratos F, Garzon-muvdi T. Quiñones-hinojosa A. A giant spinal arterial aneurysm in a child presenting as quadriparesis. BMJ Case Rep. 2013;2013.

30. Pahl FH. De oliveira MF, Rotta MA. Dias GM, Rezende AL. Rotta JM. Spontaneous resolution of an isolated cervical anterior spinal artery aneurysm after subarachnoid hemorrhage. Surg Neurol Int. 2014;5:139.

31. Gutierrez Romero D. Batista AL, Gentric JC. Raymond J, Roy D. Weill A. Ruptured isolated spinal artery aneurysms. Report of two cases and review of the literature. Interv Neuroradiol. 2014:20(6):774-80.

32. Bell DL. Stapleton CJ, Terry AR. Stone JR, Ogilvy CS. Clinical presentation and treatment considerations of a ruptured posterior spinal artery pseudoaneurysm. J Clin Neurosci. 2014;21(7):1273-6. 
33. Johnson J. Patel S, Saraf-lavi E. Aziz-sultan MA, Yavagal DR. Posterior spinal artery aneurysm rupture after 'Ecstasy' abuse. J Neurointerv Surg. 2015;7(7): e23.

34. Ronchetti G. Morales-valero SF, Lanzino G. Wald JT. A cause of atypical intracranial subarachnoid hemorrhage: posterior spinal artery aneurysms. Neurocrit Care. 2015;22(2):299-305.

35. Sung TH. Leung WK, Lai BM. Khoo JL. Isolated spinal artery aneurysm: a rare culprit of subarachnoid haemorrhage. Hong Kong Med J. 2015;21(2):179-82.

36. Takata M. Takayama M, Yokoyama Y. Hayashi H, Kishida N. An Isolated Posterior Spinal Aneurysm Resection in Which Intraoperative Electrophysiological Monitoring Was Successfully Used to Locate the Lesion and to Detect the Possibility of Ischemic Complications. Spine. 2016;41(1): E46-9.

37. Doberstein CA. Bouley A, Silver B. Morrison JF, Jayaraman MV. Ruptured aneurysms of the intradural artery of adamkiewicz: Angiographic features and treatment options. Clin Neurol Neurosurg. 2016;146:152-5.

38. Ikeda S. Takai K, Kikkawa Y, et al. Ruptured posterior spinal artery aneurysm: intraoperative and histologic findings with appreciable thrombosis. Spine J. 2016;16(3):e215-7.

39. Hill TC. Tanweer O, Thomas C, et al. Posterior Spinal Artery Aneurysm Presenting with Leukocytoclastic Vasculitis. J Cerebrovasc Endovasc Neurosurg. 2016:18(1):42-7.

40. Roka YB. Isolated cervical anterior spinal artery aneurysm: case report. $\mathrm{Br} J$ Neurosurg. 2017;:1-2.

41. Kogan M. Morr S, Siddiqui AH. Serial magnetic resonance imaging findings in subarachnoid hemorrhage due to an initially angiographically occult type II spinal aneurysm: Case report. Acta Biomed. 2017;88(1):74-8.

42. Aljuboori Z. Sharma M, Simpson J. Altstadt T. Surgical Management of Ruptured Isolated Aneurysm of Artery of Adamkiewicz: Interesting Report and Overview of Literature. World Neurosurg. 2018;11:36-40.

43. Aguilar-Salinas P. Lima J, Brasiliense LBC. Hanel RA, Sauvageau E. Ruptured aneurysm of the artery of Adamkiewicz: is conservative management the standard of treatment in the current era? J Neurointerv Surg. 2018;10(8):e22.

44. Ren Y. He M, You C. Li J. Successful Surgical Resection of Spinal Artery Aneurysms: Report of 3 Cases. World Neurosurg. 2018:109:171-8.

45. Morozumi M. Imagama S, Ando K, et al. Surgical intervention for a pediatric isolated intramedullary spinal aneurysm. Eur Spine J. 2018:27(Suppl 3):342-6.

46. Simon-Gabriel CP. Urbach H, Meckel S. Ruptured Fusiform Aneurysm of the Anterior Spinal Artery: Successful Treatment with Flow Diverter Stent Placed in the Feeding Vertebral Artery. Clin Neuroradiol. 2018;28(4):613-6.

47. Priola SM. Heyn C, Da costa L. Minimally invasive approach for removal of a ruptured radiculo-medullary artery aneurysm. Case report and literature review. World Neurosurg. 2019;126:605-10

\section{Publisher's Note}

Springer Nature remains neutral with regard to jurisdictional claims in published maps and institutional affiliations.

Ready to submit your research? Choose BMC and benefit from:

- fast, convenient online submission

- thorough peer review by experienced researchers in your field

- rapid publication on acceptance

- support for research data, including large and complex data types

- gold Open Access which fosters wider collaboration and increased citations

- maximum visibility for your research: over $100 \mathrm{M}$ website views per year

At $\mathrm{BMC}$, research is always in progress.

Learn more biomedcentral.com/submissions 